DOI: $10.2478 / \mathrm{v} 10324-012-0006-3$<smiles>CC1=CC=C1</smiles>

VERSITA
Analele Universităţii de Vest,

Timişoara

Seria Matematică - Informatică

L, 1, (2012), 77- 82

\title{
Representing a Concept Lattice by Neighborly Ideas
}

\author{
Hua Mao
}

\begin{abstract}
.
Concept lattices are an ordering of the maximal rectangles defined by a binary relation. This paper, using the idea of neighbor sets in graph theory, presents an approach to obtain all the concepts for a context, and simultaneously gets the diagram of the concept lattice.
\end{abstract}

AMS Subject Classification (2000). 05C90; 06B05; 68R10; $68 \mathrm{~T} 15$

Keywords. Concept lattice; graph; neighbor; cover; co-height

\section{Introduction}

Using neighbor idea, this paper searches the concepts and the diagram for a context. Though, there are many outcomes in concept lattice theory obtained by lattice theory and graph theory (see $[1-3,6,7,9,10]$ ), we still hope to find a new walk for neighbor's application.

First of all, we will review some knowledge what we need later on. We assume that all sets under consideration in this paper are finite. 


\subsection{Graph theoretical notions}

In this subsection we introduce some necessary graph theoretical terminologies from $[1,5]$. For more information, see $[1,5]$.

Definition 1 [1,5] A graph $G$ is a pair $(V, E)$ such that $V$ is a nonempty set and $E$ is a subset of $\mathcal{P}_{2}(V)$, the set of all two-element subsets of $V$. In a graph $G=(V, E)$, for $x \in V$, its neighbor is $N(x)=\{y \in V \mid(x, y) \in E\}$. $A$ bipartite graph (bigraph) is a graph $G=(V, E)$ for which there exists a non-trivial partition $\left\{V_{L}, V_{R}\right\}$ of $V$ such that for each $e \in E, e \cap V_{L} \neq \emptyset$ and $e \cap V_{R} \neq \emptyset$.

\subsection{Concept lattice notions}

We follow the definitions introduced in $[1,6,7]$, and repeat a few here, especially when there are graph-theoretical interpretations of interest.

Definition 2 A formal context (simply, context) is a triple $\mathbb{K}=(O, M, I)$ of nonempty set, where $I \subseteq O \times M$ is a binary relation between $O$ and $M$. For $A \subseteq O$ and $B \subseteq M, A^{\prime}=\{m \in M \mid(g, m) \in I$, for all $g \in A\}$, and $B^{\prime}=\{g \in O \mid(g, m) \in I$, for all $m \in B\} .(A, B)$ is a concept of $\mathbb{K}$ if and only if $A \subseteq O, B \subseteq M, A^{\prime}=B$ and $A=B^{\prime}$. If $\mathbb{K}$ satisfies $O \cap M=\emptyset$, then we say it is bigraph inducing. The bipartite graph $G_{\mathbb{K}}$ of a bigraph inducing formal context $\mathbb{K}=(O, M, I)$ is $G_{\mathbb{K}}=(O \cup M,\{(o, m) \mid o I m\})$.

The set of concepts of $\mathbb{K}$ is denoted by $\mathcal{B}(\mathbb{K})$ and it is called the concept lattice of $\mathbb{K}$. $[6,7]$ has the following simple rules:

$\left(A_{1}, B_{1}\right) \leq\left(A_{2}, B_{2}\right) \Leftrightarrow A_{1} \subseteq A_{2}\left(\Leftrightarrow B_{2} \subseteq B_{1}\right)$, where $\left(A_{j}, B_{j}\right) \in \mathcal{B}(\mathbb{K})$. $(\mathcal{B}(\mathbb{K}), \leq)$ is a complete lattice. After inspecting $\mathcal{B}(\mathbb{K})$, in what follows, we only need to consider the context $(O, M, I)$ satisfying $O \cap M=\emptyset,(O, \emptyset)=1$ and $(\emptyset, M)=0$, where 1 is the greatest element and 0 is the least element in $\mathcal{B}(\mathbb{K})$. Under such suppositions, $G_{\mathbb{K}}$ is an undirected simple bipartite graph. By $[1,3]$, we see that $P^{\prime}=\cap_{p \in P} N(p)$ holds for all $P \subseteq O$, dually, $B^{\prime}=$ $\cap_{a \in B} N(a)$ for $B \subseteq M$. Conversely, for a bigraph $G=\left(V_{L} \cup V_{R}, E\right)$, we define a bigraph inducing formal context $\mathbb{K}_{G}$ as $\left(V_{L}, V_{R}, I_{G}=\left\{(x, y) \mid x \in V_{L}, y \in\right.\right.$ $\left.\left.V_{R},(x, y) \in E\right\}\right)$. Hence, it is valuable to consider concept lattices with bigraph theory.

\subsection{Lattice notions}

According to $[4,8]$ : in a lattice $L, a$ covers $b$ (in notation, $b \prec a$ ) if $b<a$ and, for no $x, b<x<a$. In this paper, all the other knowledge regarding lattice theory follows $[4,8]$. We just present a definition of co-height which is not presented in $[4,8]$. 
Definition 3. For a finite lattice $L$, the co-height ch $(x)$ of $x \in L$ is defined as: $\operatorname{ch}(x)=\max \left\{n \mid x \prec x_{1} \prec \ldots \prec x_{n}=1\right\}$, where 1 is the greatest in $L$.

\section{Concept lattice}

This section presents the relationship between a concept and its covered elements in a concept lattice by the terminology of neighbor sets. Meanwhile, we may compute the concepts along with their structure, i.e. the arcs of the Hasse diagram of the lattice.

Obviously, we can not have $(X, Y) \in \mathcal{B}(\mathbb{K})$ such that $(X, Y) \prec(A, B)$ when $(A, B)=(\emptyset, M)$. We will find the necessary or sufficient conditions for $(X, Y) \in \mathcal{B}(\mathbb{K})$ covered by $(A, B) \in \mathcal{B}(\mathbb{K}) \backslash(\emptyset, M)$ in the following lemmas. In addition, though the results in Lemma $1(2)$ are familiar to the readers if they use the terms of lattice theory, we just try to prove them using the neighborly languages. Additionally, we discover the sufficient conditions for $(X, Y) \in \mathcal{B}(\mathbb{K})$ covered by $(A, B) \in \mathcal{B}(\mathbb{K})$ by the language of neighbor.

\section{Lemma 1.}

(1) Let $m_{0} \in M$ satisfy $N\left(m_{0}\right) \not \subset N(m)$ for all $m \in M \backslash m_{0}$, then $\left(N\left(m_{0}\right),\left\{m \in M \mid N(m)=N\left(m_{0}\right)\right\}\right) \in \mathcal{B}(\mathbb{K})$.

(2) Let $(A, B) \in \mathcal{B}(\mathbb{K}) \backslash(\emptyset, M)$.

(2.1) In $G_{\mathbb{K}}$, if $y_{0} \in M \backslash B$ satisfies $\emptyset \neq N\left(y_{0}\right) \cap A \not \subset N(y) \cap A$ for all $y \in M \backslash B$, then $\left(N\left(y_{0}\right) \cap A,\{y \in M \backslash B \mid N(y) \cap A=\right.$ $\left.\left.N\left(y_{0}\right) \cap A\right\} \cup B\right) \in \mathcal{B}(\mathbb{K})$, and is covered by $(A, B)$.

(2.2) If $N(y) \cap A=\emptyset$ for all $y \in M \backslash B$, then $(\emptyset, M) \prec(A, B)$ in $\mathcal{B}(\mathbb{K})$.

Proof. Routine verification.

Lemma 2. Let $(A, B) \in \mathcal{B}(\mathbb{K}) \backslash(\emptyset, M),(X, Y) \in \mathcal{B}(\mathbb{K})$ and $(X, Y) \prec(A, B)$. Then

(1) If $N(m) \cap A=\emptyset$ for all $m \in M \backslash B$, then $(X, Y)=(\emptyset, M)$.

(2) If $N\left(y_{2}\right) \cap A \neq \emptyset$ for some $y_{2} \in Y \backslash B$, then there is $y_{0} \in M \backslash B$ satisfying $(X, Y)=\left(N\left(y_{0}\right) \cap A, B \cup\left\{m \in M \backslash B \mid N(m) \cap A=N\left(y_{0}\right) \cap A\right\}\right)$. 
Proof. (1) Routine verification by a contradiction.

(2) $(X, Y) \prec(A, B)$ follows $Y=B \cup(Y \backslash B)$ and $Y \backslash B \neq \emptyset$.

$(X, Y) \in \mathcal{B}(\mathbb{K})$ leads to $Y=X^{\prime}=\{y \in M \mid \forall x \in X, x I y\}=B \cup\{y \in$ $M \backslash B \mid \forall x \in X, x I y\}$. In graphic words, we have $X \subseteq N(y)$ for all $y \in Y$. Thus, $X \subseteq N(y) \cap A$ for all $y \in Y$.

Suppose there is $y \in Y$ such that $X \neq N(y) \cap A$. This leads to $X \subset N(y) \cap A$ for some $y \in Y$. Thus, $X \subset N\left(y_{2}\right) \cap A \neq \emptyset$. Further, by Lemma 1(2), there is $y_{1} \in M \backslash B$ such that $N\left(y_{2}\right) \cap A \subseteq N\left(y_{1}\right) \cap A$ and $\left(N\left(y_{1}\right) \cap A, B \cup\{m \in\right.$ $\left.\left.M \backslash B \mid N(m) \cap A=N\left(y_{1}\right) \cap A\right\}\right) \in \mathcal{B}(\mathbb{K})$.

Evidently, we have $\left(N\left(y_{1}\right) \cap A, B \cup\left\{m \in M \backslash B \mid N\left(y_{1}\right) \cap A=N(m) \cap A\right\}\right)<$ $(A, B)$. In addition, $X \subset N\left(y_{2}\right) \cap A \subseteq N\left(y_{1}\right) \cap A$ and Definition 2 hints $(X, Y)<\left(N\left(y_{1}\right) \cap A, B \cup\left\{m \in M \backslash B \mid N\left(y_{1}\right) \cap A=N(m) \cap A\right\}\right)$. These produce a contradiction to $(X, Y) \prec(A, B)$.

We get $X=N(y) \cap A$ for some $y \in Y$.

If $y \in B$, then $X=A$ because of $(A, B) \in \mathcal{B}(\mathbb{K})$. But this is a contradiction to $(X, Y) \prec(A, B)$. Namely, $X=N\left(y_{4}\right) \cap A$ for some $y_{4} \in Y \backslash B \subseteq M \backslash B$. We assert $X \neq \emptyset$. Otherwise, we will have $N\left(y_{4}\right) \cap A \subset N\left(y_{2}\right) \cap A \subseteq$ $N\left(y_{3}\right) \cap A \neq \emptyset$ for some $y_{3} \in M \backslash B$ and $N\left(y_{3}\right) \cap A \not \subset N(y) \cap A$ for all $y \in M \backslash B$.

Lemma 1(2) implies that $y_{3} \in M \backslash B$ satisfies $(X, Y)=(\emptyset, M)<\left(N\left(y_{3}\right) \cap\right.$ $\left.A, B \cup\left\{y \in M \backslash B \mid N(y) \cap A=N\left(y_{3}\right) \cap A\right\}\right)<(A, B)$. It contradicts to $(X, Y) \prec(A, B)$.

Besides, Lemma 1(2) implies $y_{0} \in M \backslash B$ satisfying $N\left(y_{4}\right) \cap A \subseteq N\left(y_{0}\right) \cap A \not \subset$ $N(y) \cap A$ for all $y \in M \backslash B$ and $(X, Y) \leq\left(N\left(y_{0}\right) \cap A, B \cup\{y \in M \backslash B \mid N(y) \cap A=\right.$ $\left.\left.N\left(y_{0}\right) \cap A\right\}\right) \prec(A, B)$. Furthermore, we obtain $(X, Y)=\left(N\left(y_{0}\right) \cap A, B \cup\{y \in\right.$ $\left.\left.M \backslash B \mid N(y) \cap A=N\left(y_{0}\right) \cap A\right\}\right)$.

For Lemma 2(2), we continue to discuss its further properties as follows.

(I) If $N(y) \cap A=\emptyset$ for all $y \in Y \backslash B$. We will have $X=\emptyset$ in virtue of $X=\cap_{y \in Y} N(y)=\cap_{y \in Y}(N(y) \cap A)$. Therefore, $(X, Y)=(\emptyset, M)$ holds. In other words, $(X, Y) \neq(\emptyset, M)$ will follow that there is some $y \in Y \backslash B$ satisfying $N(y) \cap A \neq \emptyset$.

Additionally, under the supposition of Lemma 2(2), for all $m \in M \backslash B, N\left(y_{0}\right) \cap$ $A \not \subset N(m) \cap A$ is correct.

(II) If there is $m_{1} \in M \backslash B$ satisfying $N\left(m_{1}\right) \cap A \neq \emptyset$. Then by Lemma 1(2), there exists $m_{0} \in M \backslash B$ satisfying $\left(N\left(m_{0}\right) \cap A, B \cup\{m \in M \backslash B \mid N(m) \cap A=\right.$ $\left.\left.N\left(m_{0}\right) \cap A\right\}\right) \in \mathcal{B}(\mathbb{K}) \backslash(\emptyset, M)$ and $\left(N\left(m_{0}\right) \cap A, B \cup\{m \in M \backslash B \mid N(m) \cap A=\right.$ $\left.\left.N\left(m_{0}\right) \cap A\right\}\right) \prec(A, B)$. Thus by lattice theory, if $(X, Y) \prec(A, B)$ and there is $m_{1} \in M \backslash B$ with $N\left(m_{1}\right) \cap A \neq \emptyset$, then $(X, Y) \neq(\emptyset, M)$. Namely, there is $y_{1} \in Y \backslash B$ with $N\left(y_{1}\right) \cap A \neq \emptyset$. 
By summing up (I), (II) and Lemma 2, we have the following consequence.

Corollary 1. Let $(A, B) \in \mathcal{B}(\mathbb{K}) \backslash(\emptyset, M),(X, Y) \in \mathcal{B}(\mathbb{K})$ and $(X, Y) \prec$ $(A, B)$. If there is $y \in M \backslash B$ with $N(y) \cap A \neq \emptyset$, then there exists $y_{0} \in M \backslash B$ such that $(X, Y)=\left(N\left(y_{0}\right) \cap A, B \cup\left\{m \in M \backslash B \mid N(m) \cap A=N\left(y_{0}\right) \cap A\right\}\right)$, and additionally, for all $m \in M \backslash B$, it follows $N\left(y_{0}\right) \cap A \supseteq N(m) \cap A$.

Furthermore, we have the main result in this paper as follows.

Theorem 1. Let $(A, B) \in \mathcal{B}(\mathbb{K}) \backslash(\emptyset, M)$. The set $\mathcal{C}$ of covered elements by $(A, B)$ in $\mathcal{B}(\mathbb{K})$ is defined as follows: if for any $m \in M \backslash B$, it has $N(m) \cap A=\emptyset$, then $\mathcal{C}=\{(\emptyset, M)\}$; otherwise, $\mathcal{C}=\{(X, Y) \mid$ there is $m_{0} \in M \backslash B$ such that for any $m \in M \backslash B, N\left(m_{0}\right) \cap A \not \subset N(m) \cap A$ is correct, and besides, $\left.X=N\left(m_{0}\right) \cap A, Y=B \cup\left\{m \in M \backslash B \mid N(m) \cap A=N\left(m_{0}\right) \cap A\right\}\right\}$.

Because in $\mathcal{B}(\mathbb{K})$, the greatest element is $(O, \emptyset)$. Using Theorem 1 , we find out all the concepts covered by $(O, \emptyset)$. These covered elements have co-height 1. For $x \in \mathcal{B}(\mathbb{K})$ and $\operatorname{ch}(x)=1$, we can use Theorem 1 to get all the concepts covered by $x$. Step by step, we will obtain all the members in $\mathcal{B}(\mathbb{K})$, because in $\mathcal{B}(\mathbb{K})$ the least element is $(\emptyset, M)$ and $\operatorname{ch}(\emptyset, M)<\infty$ and any of other members has co-height less than $\operatorname{ch}(\emptyset, M)$.

We may easily know that on the efficient generation of the concepts defined by a binary relation, we compute the concepts along with their structure, i.e. the arcs of the Hasse diagram of the lattice. We will show the complexity for concept generation using our graph results. Let $|O|=n$ and $|M|=t$. For every $y \in M$, it will be generated its neighbor in linear $O(n)$ time. For per generate concept $(A, B)$, the complexity for all the elements covered by $(A, B)$ will take $O(t)$ time. Furthermore, because of $\operatorname{ch}(\mathcal{B}(\mathbb{K})) \leq n$, the complexity of our method described beyond is $O(n t)$. A.Berry et.al. in [3] give a way to generate and store all the concepts in $O\left(n^{2}\right)$ using graph theory. Also A.Berry et.al said [3] that using graph theory, some best algorithms have complexity $O(n t)$. Thus, we believe that our approach here is a better way to generate a concept lattice using graph theory.

Acknowledgment. This research is supported by Science and Technology Item of Baoding, China (11ZG005). 


\section{References}

[1] J. Abello, A. J. Pogel, and L. Miller, Breadth first search graph partitions and concept lattices, J. of Universal Computer Science, 10(8), (2004), 934-954.

[2] A. Berry, J. Bordat, and A. Sigayret, A local approach to concept generation, Annals of Mathematics and Artificial Intelligence, 49(1-4), (2007), 117-136.

[3] A. Berry and A. Sigayret, Representing a concept lattice by a graph., Dis. Appl. Math., 144, (27-42.)

[4] G. Birkhoff, Lattice Theory. 3rd. ed. Providence, American Mathematical Society, 1967.

[5] J. A. Bondy, Graph Theory with Applications, Elsevier Science Publishing Co. Inc., New York, 1976.

[6] B. A. Davey and H. A. Priestley, Introduction to Lattices and Order, 2nd. ed. Cambridge, Cambridge University Press, 2003.

[7] B. Ganter and R. Wille, Formal Concept Analysis, Mathematical Foundations Heidelberg, Springer-Verlag Berlin, 1999.

[8] G. Grätzer, General Lattice Theory. 2nd. ed. Basel, Birkhäuser Verlag, 1998.

[9] H. Mao, Concept lattices and co-covering graphs., Inter. J. of Alge., 3(3), (2009), 137-148.

[10] S. Owais, P. Gajdoš, and V. Snášel, Usage of genetic algorithm for lattice drawing, In: CLA 2005(Eds.: R. Bělohlǎvek and V. Snášel) 82-91. ISBN 80-248-0863-3.

Hua Mao

Department of Mathematics, Hebei University,

Baoding 071002, China

E-mail: yushengmao@263.net

Received: 27.07.2011

Accepted: 27.11.2011

Revised: 17.11.2011 